\section{Production of a Homozygous Mutant Embryonic Stem Cell Line (Double Knockout)}

Homozygous mutant embryonic stem (ES) cell lines have proven very useful in studying gene and protein function, and there are several reasons why this system might be preferred. Because many genes express their phenotype at the level of individual cells, using these cell lines eliminates the time and expense of producing a mutant animal. They can be isolated directly from a heterozygous mutant cell line (UNIT 23.5) without rederiving a cell line or using primary tissues from a mutant animal. In some cases, analysis of the phenotype of homozygous mutant cells can be performed more easily in culture. In addition, homozygous mutant ES cell lines may be produced even when a homozygous mutation is lethal to the animal. Finally, microinjection of homozygous mutant ES cells into blastocysts may allow investigation of the developmental potential of cells with such lethal mutations.

Selection of a homozygous cell line depends on being able to increase antibiotic concentrations such that nearly all of the cells containing a single copy of the selectable marker gene will be killed. If the wild-type neo gene is used in combination with a strong promoter (e.g., phosphoglycerate kinase; PGK), cells containing one neo gene may not be killed by the highest $\mathrm{G} 418$ concentrations that can be obtained. For this technique to be effective, therefore, it is recommended that a mutant neo gene containing a point mutation that decreases phosphotransferase activity be used in place of wild-type neo (Yenofsky et al., 1990). This mutant neo gene is readily available because it is contained in some versions of both pMC1-neo and $p_{\mathrm{PGK}}-n e o$ (see Fig. 23.5.1). The wild-type hygromycin-B-phosphotransferase $(h y g)$ gene used in combination with the PGK promoter, $p_{\mathrm{PGK}}-h y g$, has also worked well in the method described in the basic protocol.

Alternatively, using a construct with loxP sites flanking the neo marker, such as those derived from pTKLNL or pTKLNCL, allows the neo to be removed from both alleles simultaneously after homozygous cells are obtained. Using this strategy, one can insert a wild-type neo to inactivate a gene of interest and still use high-G418 selection to isolate homozygous cells.

\section{SELECTION FOR HOMOZYGOUS CLONES}

In this protocol, a homozygous mutant ES cell line is isolated from heterozygous mutant ES cells (UNIT 23.5) by culturing the cells in increasing concentrations of G418. The more stringent selection conditions favor cells that contain, as a result of the loss of heterozygosity, two selectable marker genes (e.g., for neo), and therefore two mutant alleles of the target gene. Resulting colonies are harvested and screened by northern hybridization or immunoblotting to confirm that the target gene is inactive.

\section{Materials}

Heterozygous mutant ES cell line, frozen in liquid nitrogen (UNIT 23.5)

ES/LIF medium (UNIT 23.5)

G418 (UNIT 9.5)

100-mm tissue culture plates, gelatin coated (UNIT 23.5)

Additional reagents and equipment for recovery of frozen cell lines (UNIT 11.9), ES cell culture (UNIT 23.5), northern analysis (UNIT 4.9), and immunoblotting (UNIT 10.8)

NOTE: All tissue culture incubations should be performed in a humidified $37^{\circ} \mathrm{C}, 5 \% \mathrm{CO}_{2}$ incubator unless otherwise noted.

Contributed by Richard Mortensen

Current Protocols in Molecular Biology (2000) 23.6.1-23.6.3

Copyright $₫ 2000$ by John Wiley \& Sons, Inc.
BASIC PROTOCOL

Manipulating the Mouse Genome 
1. Thaw heterozygous mutant ES cells. Culture in ES/LIF medium (UNIT 23.5), passaging cells every 2 to 3 days by seeding a 100-mm gelatin-coated tissue culture plate with $1-2 \times 10^{6}$ cells/plate.

Selection should be performed with more than one heterozygous mutant ES cell line clone because the efficiency of conversion to homozygosity varies among cell lines. In addition, more than one clone should be analyzed for the phenotype.

2. Begin the selection by plating three $100-\mathrm{mm}$ gelatin-coated tissue culture plates at $10^{5}$ cells/plate using ES/LIF medium and adding $1.0,1.5$, or $2.0 \mathrm{mg} / \mathrm{ml} \mathrm{G} 418$ (final), respectively.

It is important to neutralize the G418 to $\sim p H$ 7.4.

Both the neo and hyg genes have been used successfully as selectable markers in mutant ES cells (R.M., unpub. observ.). To select for hyg, use ES/LIF medium containing 1.0, 1.5, or $2.0 \mathrm{mg} / \mathrm{ml}$ hygromycin-B (HPH; UNIT 9.5).

3. Incubate cells 7 to 10 days. Change the medium each day, using ES/LIF medium with the appropriate concentration of G418 added, until single colonies are detected.

If cells overgrow plates and no single colonies are obtained, repeat steps 2 and 3 using a higher $\mathrm{G} 418$ concentration (e.g., 2, 4, or $6 \mathrm{mg} / \mathrm{ml}$ final).

4. Screen colonies for homologous recombinants as described in UNIT 23.5 (Basic Protocol, steps 15 to 21 ).

The hybridizing fragment indicative of the unaltered target gene will be completely absent in homozygous mutant cells. Typically, 50\% of the clones are homozygous but because the process is random, individual heterozygous clones may yield homozygous clones at a frequency of $4 \%$ to $100 \%$.

5. Perform a northern hybridization analysis (UNIT 4.9) of mRNA or an immunoblot analysis of protein (UNIT 10.8) to confirm that the target gene is inactive. No normal mRNA or protein should be found.

6. If the constructs contain loxP sites flanking the neo gene, remove the neo by transient expression of Cre (see UNIT 23.5, Support Protocol).

Both copies of neo are removed with same efficiency as one copy.

\section{COMMENTARY}

\section{Background Information}

Comparison of the phenotype of wild-type and homozygous mutant cells should provide information concerning the function of the targeted gene, provided that the gene functions in the cell assayed. ES cells offer a unique opportunity because they are capable of differentiating into any cell type. Many cell types can be produced through manipulation of in vitro cultures, including beating cardiocytes, skeletal muscle cells, neurons, glial cells, and vascular endothelial cells. Provided that the phenotype can be analyzed with a single cell or with a few cells, these differentiated cells can then be used to analyze the phenotype of the disrupted gene.

Because the normal number of chromosomes is maintained in ES cells (Mortensen et al., 1992), this method should be applicable to the study of gene function in development. ES cells can also differentiate within the entire organism when injected into normal blastocysts. Homozygous mutant cells offer no technical advantage over heterozygous cells if the goal is to obtain a mutant mouse line through germline transmission; however, if homozygous mutant cells are tagged by introducing a gene that has a histochemically detectable product (e.g., $\beta$-galactosidase), then the fate of the homozygous mutant cells in the animal can be determined easily. Studies of embryos derived from blastocysts injected with tagged mutant ES cells may reveal the role of the target gene during development even if the mutant gene is lethal for the animal when homozygous.

In some cases, the phenotype of the disrupted gene may be tested by introducing heterologous proteins into the cultured cells. For example, an intracellular signaling pathway 
may be investigated by expression of the particular receptor that creates an intracellular response to an applied agonist.

Cells produced using this protocol are only resistant to neo and its analogs. Thus, further genetic manipulations of the cells, such as the targeting of other genes using homologous recombination, can be performed using other drug selections (e.g., HPH; UNIT 9.5).

The suitability of this technique for the production of homozygous mutant cell lines other than ES cells is less certain. Homologous recombination and spontaneous loss of heterozygosity occur in some other cell lines and these same methods have been used to produce homozygous mutant cell lines; however, the methods may not be widely applicable, as other types of immortalized cells (e.g., lymphocytes or fibroblasts) may not undergo homologous recombination of a targeting construct at the same frequency as ES cells. Furthermore, many immortalized cells are markedly polyploid or aneuploid. If more than two copies of the target gene are present, this method for producing homozygous mutant cells may not be suitable; however, because these cells often lose or gain chromosomes, some clones might be isolated with no endogenous gene remaining.

The loss of heterozygosity seems to occur by several different mechanisms that vary with cell type. The mechanism by which this conversion occurs is unknown. Some possibilities include nondisjunction, chromosomal loss and duplication, or gene conversion.

\section{Critical Parameters and Troubleshooting}

Homozygous mutant ES cells will not be obtained if the mutation is lethal for the ES cell. The critical technical factor in this method is determining the amount of G418 needed to select homozygous over heterozygous cells. Differences in G418 levels required for different constructs can arise from two sources: the strength of the promoter and the influence of the genomic site on neo expression. In addition, the resistance to G418 may vary, depending on the enzymatic activity of a wild-type or mutant neo gene (Yenofsky et al., 1990). If the heterozygous mutant ES cells are resistant to the highest concentration of antibiotic (i.e., 2 $\mathrm{mg} / \mathrm{ml} \mathrm{G} 418$ ), the G418 concentration should be increased (e.g., up to $6 \mathrm{mg} / \mathrm{ml}$ ). The endpoint should be survival of individual colonies with death of nearly all cells.

It should be noted that this selection method allows only a minority of the homozygous clones to survive. In one case, homozygous mutant cells constituted $25 \%$ of the cells (presumably due to the chance conversion of a heterozygous to homozygous cell early in the life of the clone). Nevertheless, $<1 \%$ of the cells survived $1 \mathrm{mg} / \mathrm{ml} \mathrm{G} 418$ (R.M., unpub. observ.).

It is also possible that loss of heterozygosity may not occur for all genomic sites, although no such genomic sites have yet been identified. Because the inability to isolate homozygous cells may lead to the erroneous conclusion that a homozygous mutation in a gene is lethal, the lethality of the mutation should be verified. A rescue experiment in which a third copy of the gene (or expressed cDNA) is introduced into the heterozygous mutant cells, followed by selection of the homozygous mutant, would distinguish between lethality and no loss of heterozygosity.

An alternative method for producing homozygous mutant cells is to disrupt the second allele of the target gene using a second targeting construct that uses a different positive selectable marker. For example, if neo was used in the first-round construct, the hyg gene can be used and cells selected in HPH as described in UNIT 23.5.

\section{Anticipated Results}

The hybridizing fragment indicative of the altered gene will be absent in homozygous mutant cells. Typically, $50 \%$ of the clones are homozygous, but because the process is random, individual heterozygous clones may yield homozygous clones at a frequency of $4 \%$ to $100 \%$.

\section{Time Considerations}

Expanding and selecting clones requires $\sim 2$ to 3 weeks. Screening clones takes several days.

\section{Literature Cited}

Mortensen, R.M., Conner, D.A., Chao, S., Geisterfer, L.A., and Seidman, J.G. 1992. Production of homozygous mutant ES cells with a single targeting construct. Mol. Cell. Biol. 12:2391-2395.

Yenofsky, R.L., Fine, M., and Pellow, J.W. 1990. A mutant neomycin phosphotransferase II gene reduces the resistance of transformants to antibiotic selection pressure. Proc. Nat. Acad. Sci. U.S.A. 87:3435-3439.

Contributed by Richard Mortensen University of Michigan Medial School Ann Arbor, Michigan
Manipulating the Mouse Genome

\subsection{3}

Supplement 52 